\title{
Fish as a source of acoustic signal measurement in an aquaculture tank: Acoustic sensor based time frequency analysis
}

\author{
Shahbaz Gul Hassan ${ }^{1,2,3}$, Shakeel Ahmad ${ }^{4}$, Shafqat Iqbal ${ }^{4}$, Ehsan Elahi ${ }^{7}$, Murtaza Hasan ${ }^{5}$, \\ Daoliang Liं ${ }^{8}$, Zhiyan Zhou ${ }^{1,2,3^{*}}$, Adnan Abbas $^{6}$, Cancan Song ${ }^{1,2,3}$ \\ (1. College of Engineering, South China Agricultural University/Guangdong Engineering Research Center for Agricultural Aviation \\ Application (ERCAAA), Guangzhou 510642, China; \\ 2. National Joint Research Center for Precision Agricultural Aviation Application Technology, Guangzhou 510642, China; \\ 3. Key Laboratory of Key Technology on Agricultural Machine and Equipment (South China Agricultural University), \\ Ministry of Education, Guangzhou 510642, China; \\ 4. College of Economics and Statistics, Guangzhou University, Guangzhou 100083, China; 5. Department of Biochemistry Biotechnology, \\ Islamia University Bahawalpur Pakistan; 6. College of Engineering, China Agricultural University, Beijing 100083, China; \\ 7. College of Economics and Management, Nanjing University of Information Science and Technology, Nanjing 210044, China; \\ 8. College of Information and Electrical Engineering, China Agricultural University, Beijing 100083, China)
}

\begin{abstract}
Acoustic signals travels rapidly in water without attenuating fish telemetry. The digital sonar and passive acoustic has been used for fish monitoring and fish feeding. However, it is an urgent need to introduce new techniques in order to monitor the growth rate of fish during harvesting and without causing adverse effects to the harvested fish. Therefore, a novel technique was introduced to probe the acoustic signal frequency ratio in absence and presence of the fish in tanks, which basically uses an acoustic sensor (hydrophone), acoustic signal processing system (scope meter), and a signal monitoring system (fluke view). Acoustic signals were selected from $48-52 \mathrm{~Hz}$ frequency, measure of dispersion of frequency signal represented as a function of time via Xlstat software. Measure of dispersion displayed a significant effect of acoustic signal in the presence and absence of the fish in tanks. These optimised protocols of this study will help to control and prevent excessive wastage of feed and enhance proper utilization of feed that chiefly enhance fish growth in aquaculture
\end{abstract}

Keywords: acoustic signals, acoustic sensor, fish, aquaculture, time frequency analysis, signal processing

DOI: $10.25165 /$ j.ijabe.20191203.4238

Citation: Hassan S G, Ahmed S, Iqbal S, Elahi E, Hasan M, Li D L, et al. Fish as a source of acoustic signal measurement in an aquaculture tank: Acoustic sensor based time frequency analysis. Int J Agric \& Biol Eng, 2019; 12(3): 110-117.

\section{Introduction}

In 2016, the contribution of aquaculture to the world's total fish production reached $47.1 \%$ compared to only $31.6 \%$ in the previous decade as obtained from statistical records from fisheries and aquaculture resources ${ }^{[1]}$. Proper fish growth monitoring is necessary for managing aquaculture, as direct fish growth monitoring effects fish feed costs which is $40 \%$ of the total cost production for the industry ${ }^{[2]}$.

Complicated intensive aquaculture with water turbidity and densities make it difficult to monitor fish growth, which greatly affect the diagnostics for proper food or feeding strategies. In this context, pollution and growth becomes management problems

\section{Received date: 2018-04-08 Accepted date: 2019-01-01}

Biographies: Shahbaz Gul Hassan, PhD, research interests: sensor design and image analysis, Email: mhasan387@cau.edu.cn; Shakeel Ahmad, PhD, research interests: statistical modeling, shakeelpk@e.gzhu.edu.cn; Shafqat Iqbal, PhD, research interests: time series, Email: shafqat905@e.gzhu.edu.cn; Ehsan Elahi, $\mathrm{PhD}$, research interests: microeconomics, econometrics, Email: ehsanelashi@ nuist.edu.cn; Murtaza Hasan, $\mathrm{PhD}$, research interests: nano technology, Email: murtazapirachiub@gmail.com; Daoliang Li, PhD, research interests: sensor, computer vison, internet of things. Email: dliangl@cau.edu.cn; Adnan Abbas, Email: dr_adnan@cau.edu.cn; Cancan Song, Master student, research interests: precision agriculture and agricultural aviation, Email: 737448145@qq.com.

* Corresponding author: Zhiyan Zhou, Professor, research interests: agricultural aviation. College of Engineering, South China Agricultural University, Guangzhou 510642, China. Tel: +86-13560026139, Email: zyzhou@scau.edu.cn. when there is overfeeding or less feeding strategy applied. To avoid these problems, many applications have been developed by researchers to control and overcome different parameters. One of them is based on mathematical models for fish feeding optimization in marine farms ${ }^{[3]}$. Allometry has been used to estimate fish growth by fish imaging technique. This method provides flexibility to optimise fish feed without extracting the fish from the tanks for weighing ${ }^{[4]}$. Depomod model provides a better predictive ability for fish growth, farm assessment and also in predicting residual accumulation ${ }^{[5]}$.

Some models presented in recent years are: Ferreira et al. ${ }^{[6]}$ proposed the Farm Aquaculture Resource Management (FARM) to determine production and eutrophication by integrating biochemical and physical models for the assessment of a fish farm productivity, but the turbulent and time varying force is not included in this model, which is considered as an import parameter and that have distinguishing effect ${ }^{[7]}$. Corner et al ${ }^{[8]}$ developed feed loss based on a Geographical information system (GIS) for feed loading sourcing many information systems in marine culture, which indicates the importance of feed loss and aquaculture environment which is important in this system for the proper propagation of acoustic signals. McCauslands et al. ${ }^{[9]}$ developed a model to simulate the interaction of tradition fishing and physical environment of aquaculture. All methods presented above discuss the improvement of aquaculture production in term of better utilization of available resources and improve the efficiency by integrating the different technological physical and environmental 
factors including software development, sensor, and water management respectively.

Different software applications have been developed to increase sustainability and production in this industry. For example, $\mathrm{In}^{[10]}$ development for economic and social sustainability, Medveg $^{[11]}$ developed for nutrients release in ecosystem, Meramed $^{[12]}$ for environment effect like temperature, salinity, turbidity etc, ICES ${ }^{[13]}$ for marine culture, Ecasa for sustainable aquaculture for water quality, socio-economics aspects ${ }^{[14]}$. All about software or systems developed in recent past are used to sustain the aquaculture economically, technically and functionally because of complex environment of aquaculture. To be best of our knowledge there are only few studies given below, which presents the importance of acoustic for the benefit of aquaculture production.

Acoustic signals travel very rapidly in water as compared to air without attenuation. Thus, acoustic signals provide a potential source of communication ${ }^{[15,16]}$. Fish have been used as source of sound production in water through their movement. In aquaculture, acoustic techniques have been applied for many years to monitor aquatic animal behaviour, to estimate their density and quantity. Acoustic monitoring systems are commonly used for fish monitoring in marine and freshwater environments. Digital scanning sonar has been used for fish feed monitoring ${ }^{[17,18]}$, Acoustic technology has been used in fish farming industry for feed monitoring and estimating fish biomass ${ }^{[19]}$. In techniques like multi-frequency and echo sounding have been used for stock assessment not real time assessment, Acoustic telemetry has been used for many years to measure heart rate, muscle activity, fish movement and vertical distribution ${ }^{[20,21]}$. However, all these methods need either the presence of humans or physical contact of the device attached to the fish ${ }^{[22]}$. To measure the fish presence quantitatively in a tank without harming the animals is not only a challenging task but also an upcoming research interest for Aquaculture $^{[23]}$.

Herein a simple and novel acoustic method presented to estimate fish biomass and growth rate using signal processing techniques and statistical comparisons. This study results showed that fish biomass and movement in tanks can be used as a proxy to measure fish growth. This technique is based on acoustic measurement of frequency in $\mathrm{Hz}$, which is directly proportional to the fish movement and biomass. These calculated measurements can be used to differentiate the total fish mass density in a tank under laboratory conditions. This study results showed that fish biomass can be used as a source of acoustic measurement which is directly related to fish growth.

In summary, this research presents the following contributions: 1) A simple and novel approach to estimate fish biomass and movement from acoustic signals; 2) A simple time series comparison to differentiate the frequency of acoustic signal in presence and absence of fish in tanks; 3) An estimative assessment of fish growth in term of frequency of the acoustic signal non-invasively to the fish.

\section{Materials and methods}

\subsection{Experiment design}

The experiment was performed in laboratory environment (College of Information and Electrical Engineering, China agricultural University, Beijing) in between March and May 2016, with water temperature from 15 to $20 \mathrm{C}$. Firstly, frequency was measured by immersing the sensor into the different places in absence of fish, later measure the frequency in presence of fish in tank. Then, compared the signal differences in absence and presence of fish under similar conditions. As in a laboratory environment signals variation depends upon the fish movement and sometimes frequency signals become very low. For this purpose, compared the cumulative distinguished frequency signals in absence and presence of fish in a tank. For each sequence, frequency signal within range of 48-52 Hz. The measurement unit consisted of hydrophone (data acquisition) connect with signal processing device, connected to computer (data storage). A metallic rod was suspended, which was used to hang the hydrophone wire.

To characterize and monitor the fish activity based on acoustical measurement ${ }^{[24,25]}$ to measure total scattering of the fish swimming in a tank by multiple recordings of reverberation time series. The accuracy of the technique to measure total scattering has been evaluated using standard metal spheres ${ }^{[26]}$, and scattering was successfully measured for krill ${ }^{[27,28]}$, fish, and humans in different environments, from air to seawater and audible to ultrasonic. All these experiments were conducted by transmitting pulses using single of multiple emitter to get reverberation. While, in present study reverberation was related directly to water movement, which depend on fish density and movement.

This experiment was performed in a tank of $3 \mathrm{~m}^{3}$. A highly sensitive hydrophone Tc4301 Version: B108 070711/USin 4 corners as well in the centre of rectangular tank to measure the frequency at different places.

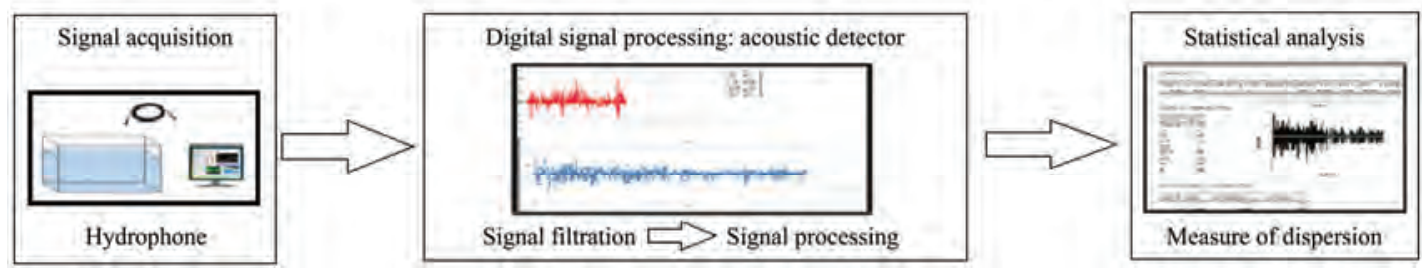

Figure 1 Presents the architecture of proposed acoustic system which includes the components: hydrophone, signal processing method and data analysis using statistical techniques

\subsection{Data acquisition}

\subsubsection{Signal filtration and Processing:}

For acoustic frequency data gathering and processing, A digital signal processing technology (Analog device, 190 II series Scope Meter Oscilloscope (model 190-202, Washington Dc, United States), software Fluke view software (Version 1.0, Washington Dc, United States) were used. The acoustic sensor was connected to analogue device 190 II series Scope Meter, which shows and records the frequency signals, the fluke view software installed in Pc and connected to 190 II series Scope Meter to monitor the frequency signals. Data was copied and stored directly from monitoring screen and saved in a portable storing device.

\subsection{Statistical analysis}

\subsubsection{Machine learning modelling}

Latterly, statistically process the gathered data, using Bayesian regression model, where $\mathrm{R}$ language and MCMCregress packages 
were used to implement this model. The data and priors were used as input which further display the generated results as a posterior distribution, that describes the posterior distribution of linear regression model. In the results obtained from the fluke view, each of the sample $(n=1200)$ with the frequency fluctuation of $(5 \mathrm{~min} / \mathrm{div})$ were used as input to Bayesian regression model. This model is tested, provided with a changing input as in absence and presence of fish of $5000 \mathrm{~g}$ and $10000 \mathrm{~g}$, respectively.

This clearly exhibit the significance of those frequency signals, generated in presence and absence of fish in tank. This paper predominantly compares the significance of frequency signals in a fish tank.

Consequently, two measurements were required for computing the significance of frequency signals: 1) Acoustic measurement of frequency signals in absence of fish, 2) Acoustic measurement of frequency signals in presence of fish. Then, Comparison of frequency signals in different aquatic density.

After model processing the RMSE (root mean square error) was calculated using following the formula:

$$
\operatorname{RMSE}=\sqrt{\sum_{i}^{n}\left(X_{o b s, i}^{n}-X_{\bmod e l, i}^{n}\right) / n}
$$

In order to distinguish the results more clearly, the results were compared using Receiver operating characteristics (ROC), which classify the binary parameters $(1,0)$ as the comparison of presence and absence of fish.

\subsubsection{Measure of Dispersion}

Later, Dispersion (standard deviation) were measured for the accumulative frequency to show the significance of using xlstat for windows, and show the significance effect of fish biomass within the tank.

$$
\text { M.O.D }=\sum_{f=1}^{n}(f-F) / n
$$

where, M.O.D is measure of dispersion; $f$ is the frequency at certain time; $F$ denotes total number of Frequency, and $\mathrm{n}$ is the number of

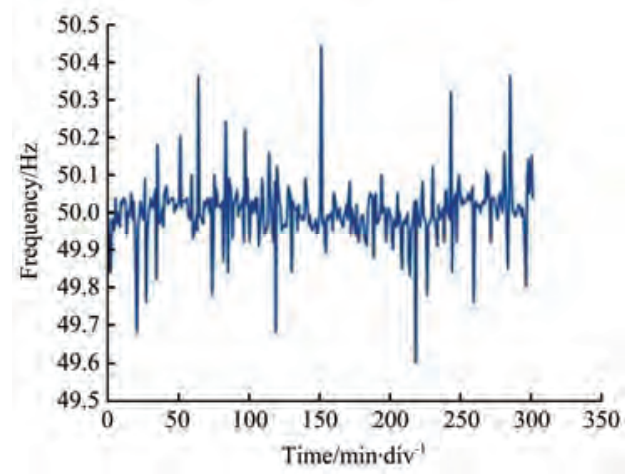

a. Presents time frequency signal in presence of fish when sensor is place on right front of tank

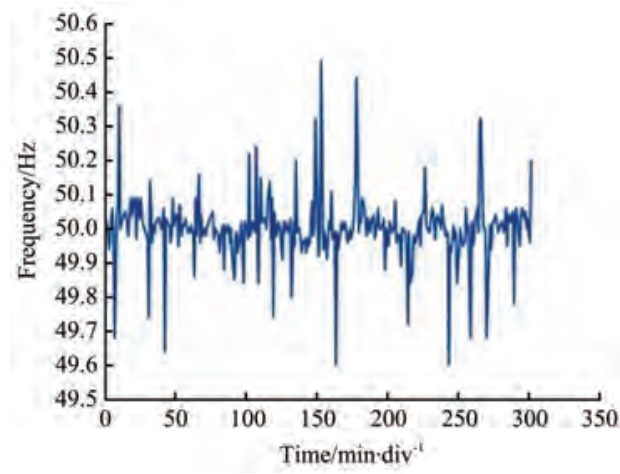

c. Presents time frequency signal in presence of fish when sensor is place on left front of tank frequency.

In a graphical term,

$$
\text { Area of Graph is }=\int_{t=0}^{t=n} f
$$

where, $t=0$ is the start time $t=n$ is end time $f$ is frequency

2.3.3 Use of Acoustic sensor (Hydrophone)

After immersing the acoustic sensor inside the tank, signals observed through fluke view software on computer screen as illustrated in Figure 3. This process repeated by immersing the acoustic sensor in different places within the tank, in the absence and presence of fish. For each interval of getting frequency fluctuations, frequency as a function of time was plotted using Fluke view attached to analogue device which collects all responses through the acoustic sensor. Although, at some stages, very low frequency fluctuations observed, as it is quite difficult for the naked eyes to differentiate between the fluctuations for both processes. But through statistically comparison among these frequency values were able to see the clear significance.

\section{Results and discussion}

The accurate detection of acoustic signals is a challenging task in critical component for any intelligent aquaculture system within limited resources. Signals frequency were received via hydrophone (Tc4013, frequency range: $1 \mathrm{~Hz}$ to $170 \mathrm{kHz}$, Teledyne Reson) immersed in the fish tank.

This Acoustic technique is widely used in aquaculture, which includes self-feeders ${ }^{[29,30]}$, acoustic monitoring on feeding practices $^{[31]}$, non-consumed pellets ${ }^{[32,33]}$, and measurement of sound generated by fish ${ }^{[34]}$. This technique is based on fish motility that produces effects on water surface and generate signals. The comparison of signals in the presence and absence of fish is presented. The measurements are not limited to any specific kind of species, but for all fish farm environment.

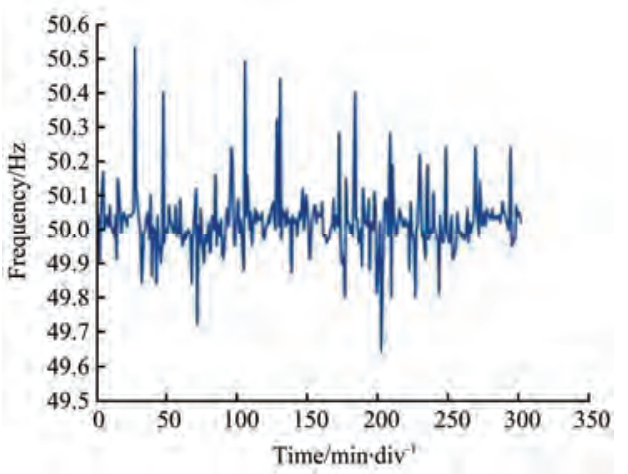

b. Presents time frequency signal in presence of fish when sensor is place on right back of tank

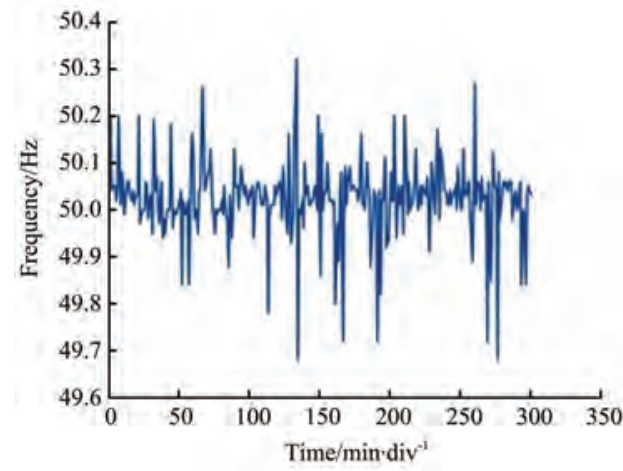

d. Presents time frequency signal in presence of fish when sensor is place on left back of tank

Figure 2 Time frequency analysis of each time displacement of sensor in presence of fish with biomass $5000 \mathrm{~g}$ 


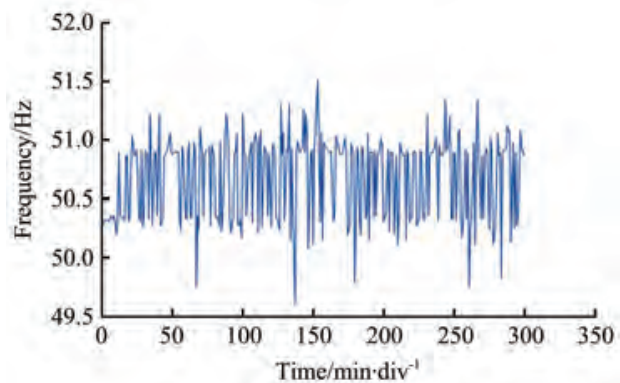

a. Presents time frequency signal in presence of fish when sensor is place on right front of tank

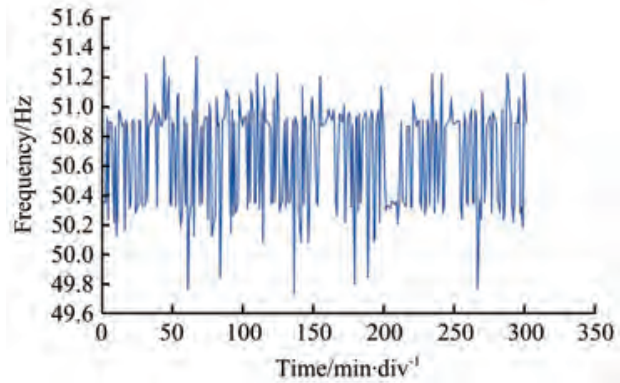

c. Presents time frequency signal in presence of fish when sensor is place on left front of tank

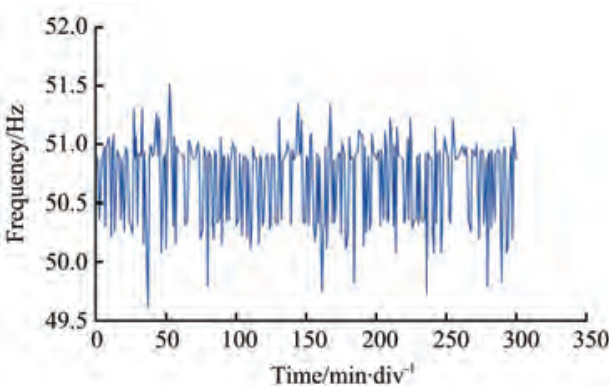

b. Presents time frequency signal in presence of fish when sensor is place on right back of tank

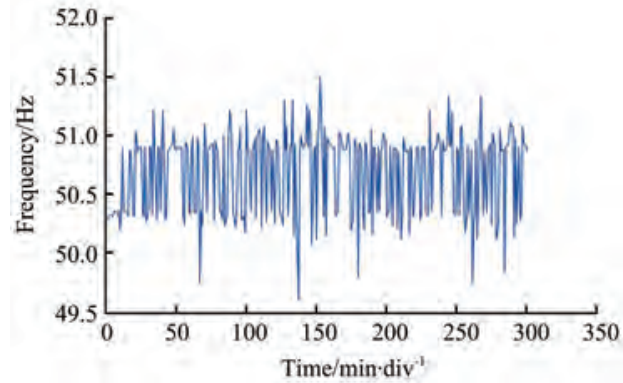

d. Presents time frequency signal in presence of fish when sensor is place on left back of tank

Figure 3 Time frequency analysis of each time displacement of sensor in presence of fish with biomass $10000 \mathrm{~g}$

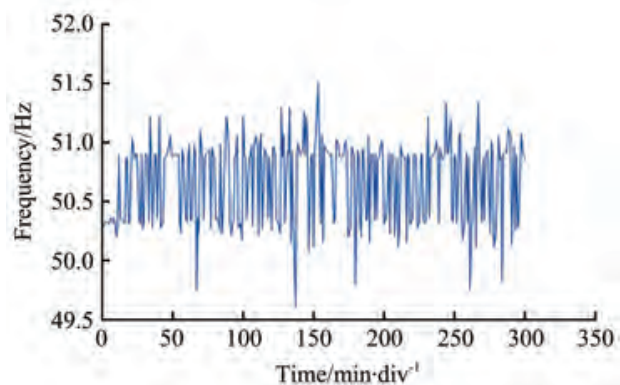

a. Presents time frequency signal in presence of fish when sensor is place on right front of tank

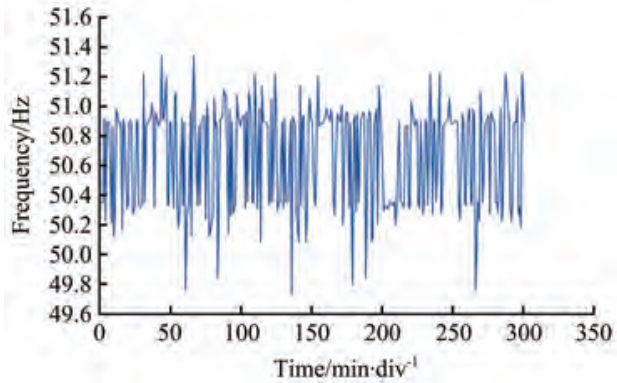

c. Presents time frequency signal in presence of fish when sensor is place on left front of tank

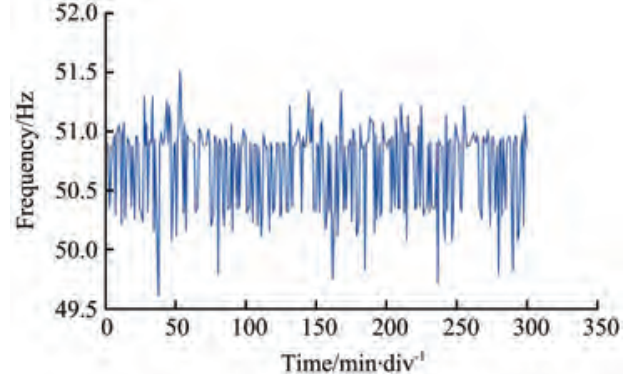

b. Presents time frequency signal in presence of fish when sensor is place on right back of tank

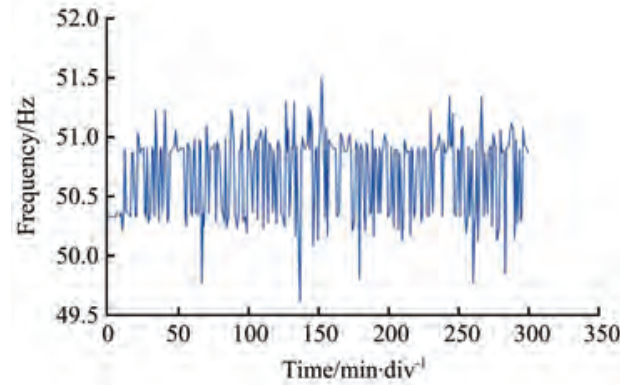

d. Presents time frequency signal in presence of fish when sensor is place on left back of tank

Figure 4 Time frequency analysis of each time displacement of sensor in absence of fish

In Figure 1 presented an example in graph by immersing the sensor into the tank; it takes five min for each division $(5 \mathrm{~m} / \mathrm{div})$. Indeed, all propagating sound in water has both pressure and particle motion components, but after some distance, often defined as the point at a distance of wavelength of the sound divided by 2 pi $(\lambda / 2 \pi)$, the pressure component of the signal dominates, though particle motion is still present and potentially important for fish $^{[35,36]}$. Therefore, frequency signals were measure by immersing the sensor at 4 different places; latterly, merged these signals for single process (presence or absence of fish).

\subsection{Comparison of Signals Measure of Dispersion}

Significance comparison is an important metric for any signal processing system in frequency and time domain that is shown graphically and statistically by comparing the measured dispersion. The presence and absence of fish in tank had shown significant effect on signal variation as show in Table 1. Consequently, the other factors (bubbling, temperature sensor, etc) were ignored. The comparison made between measure of dispersion were proven significant, which is due to presence of fish movement. To illustrate the effectiveness of signal comparison is shown graphically. Each point of signal detection is shown in in Figure 4 Finally, cumulative signal detection displayed as shown graphically in Figure 5, Figure 5a and Figure 6 that is in the presence of fish with biomass density $5000 \mathrm{~g}, 10000 \mathrm{~g}$ and absence $(0 \mathrm{~g})$ of fish respectively. Along with the displayed comparison of measure of dispersion in Table 1. 


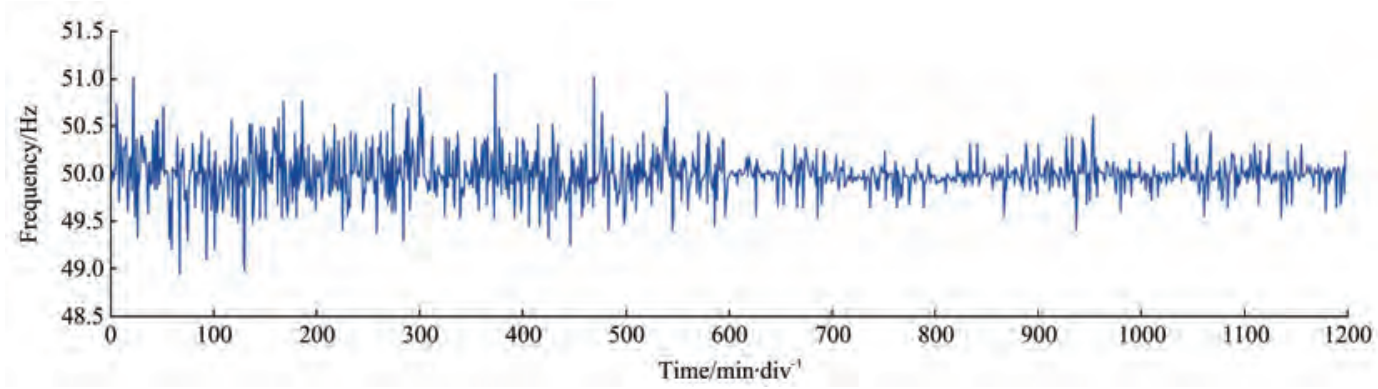

Figure 5 Time frequency analysis for cumulative signals in fish presence with biomass density $5000 \mathrm{~g}$

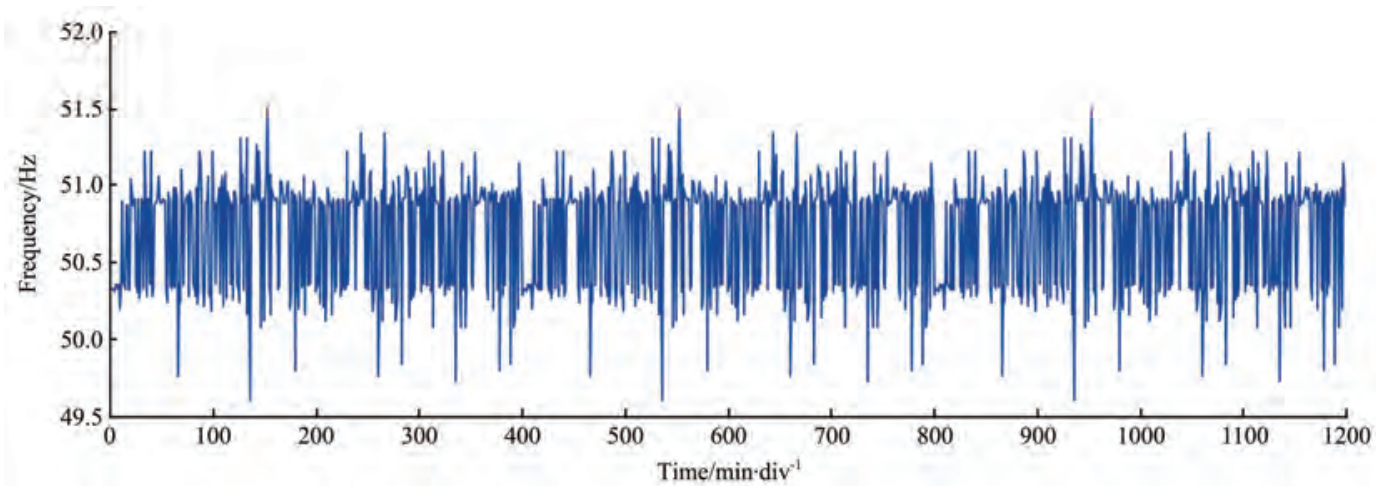

Figure 6 Time frequency analysis for cumulative signals in fish presence with biomass density $10000 \mathrm{~g}$

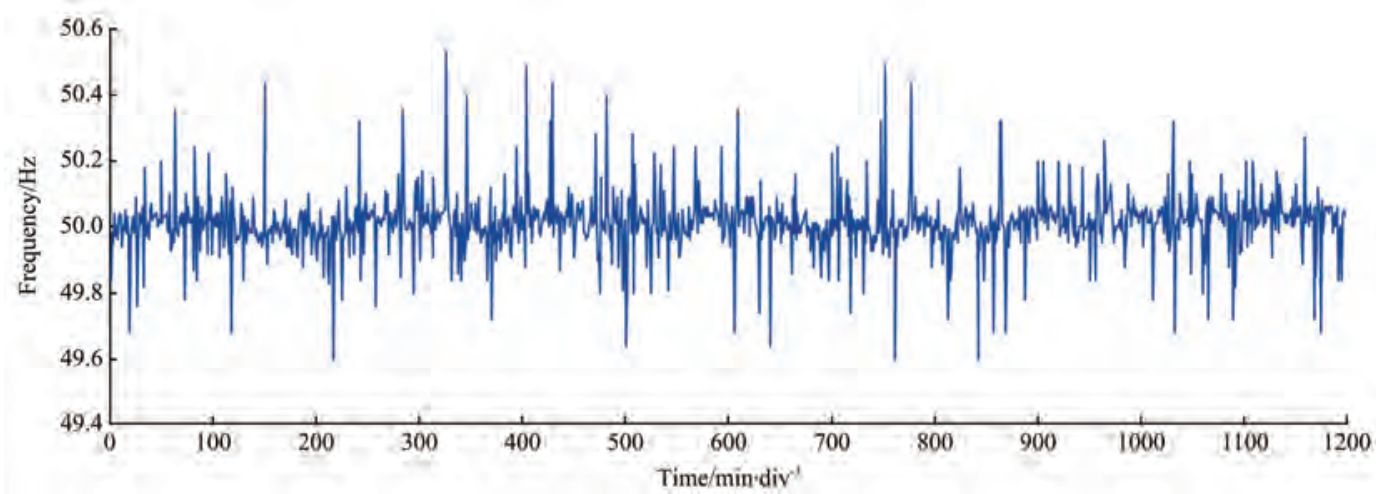

Figure 7 Time frequency analysis for cumulative signals in absence of fish

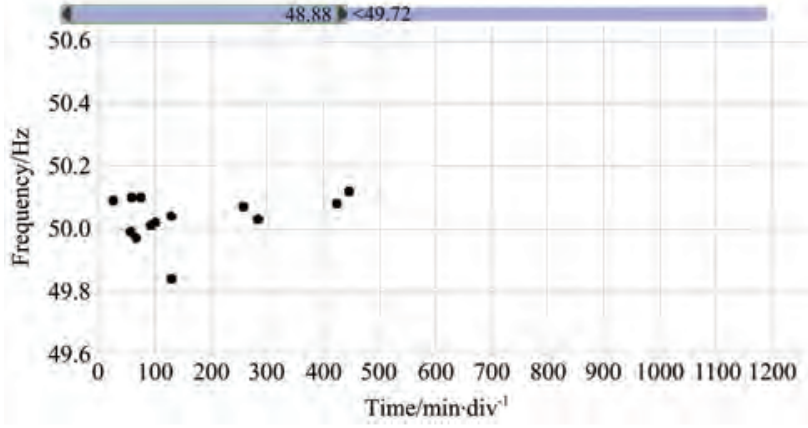

a. Presents time frequency signal in absence of fish in range 49.9-49.88

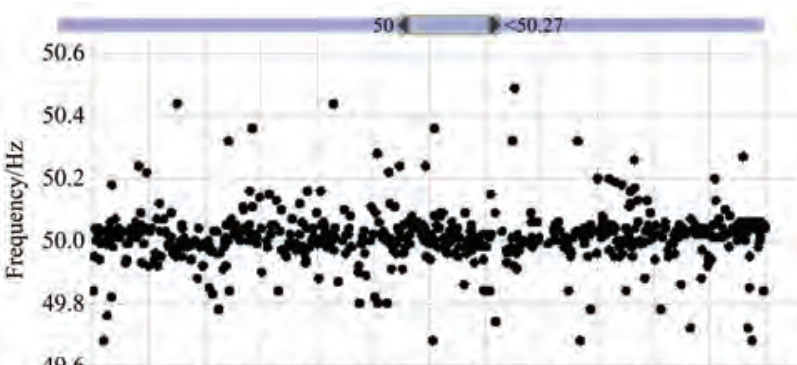

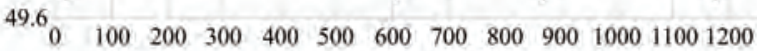
Time/min'div

c. Presents time frequency signal in absence of in range 50.03-50.18

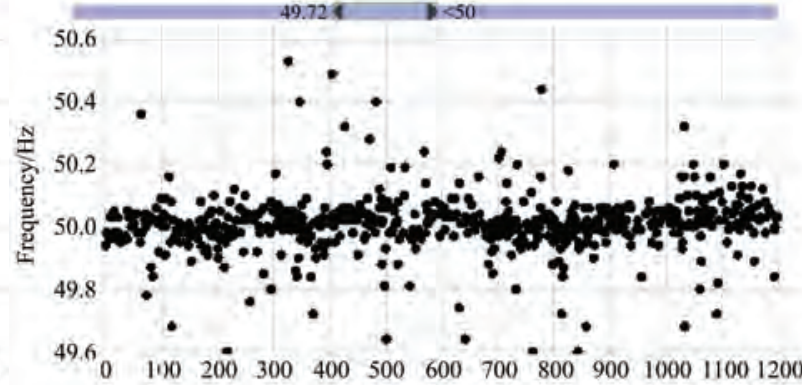

Time/min $\cdot \operatorname{div}^{-1}$

b. Presents time frequency signal in absence of fish in range 49.88-50.03

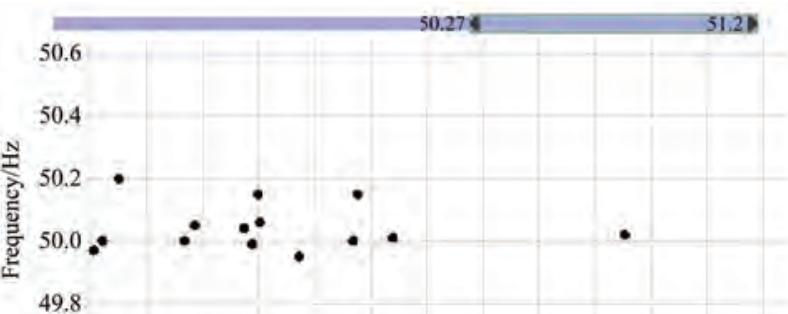

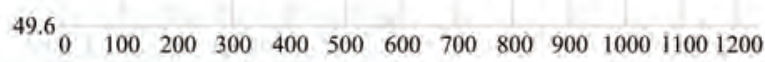
Time/min'div ${ }^{-1}$

d. Presents time frequency signal in absence of fish in range 50.18-50-60

Figure 8 Signal frequency dispersion in different range in absence of fish 


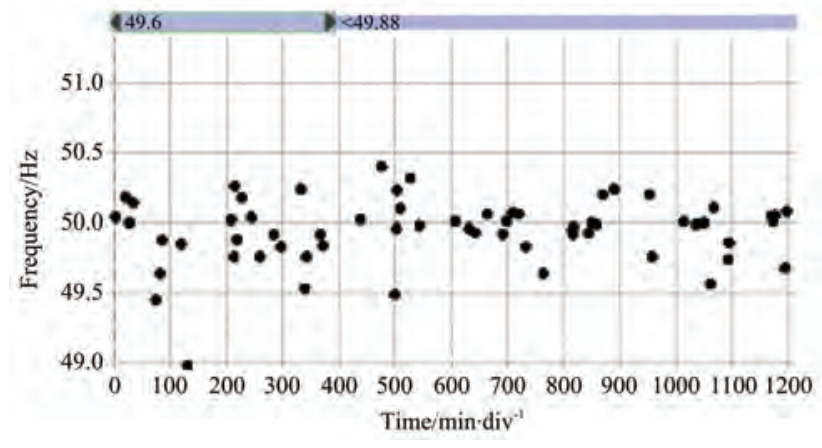

a. Presents time frequency signal in presence of fish in the ranges 48.88-49.72

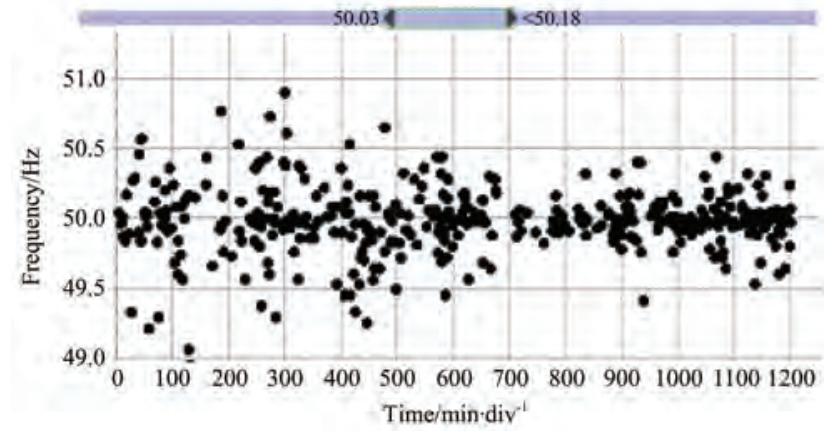

c. Presents time frequency signal in presence of in the ranges 50-50.27

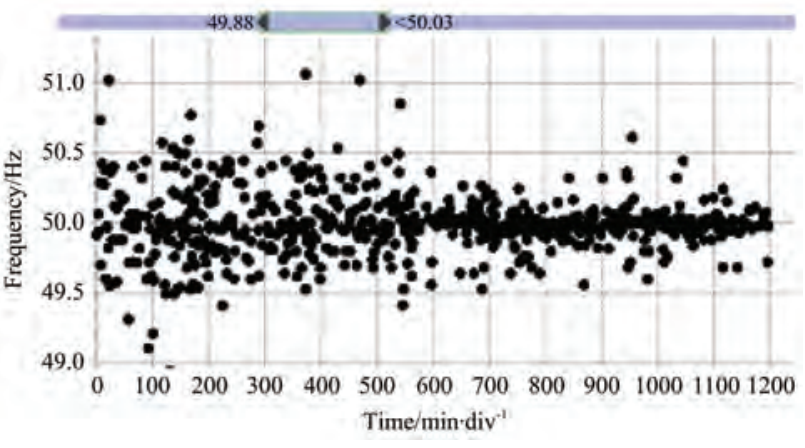

b. Presents time frequency signal in presence of fish in the ranges $49.72-50$

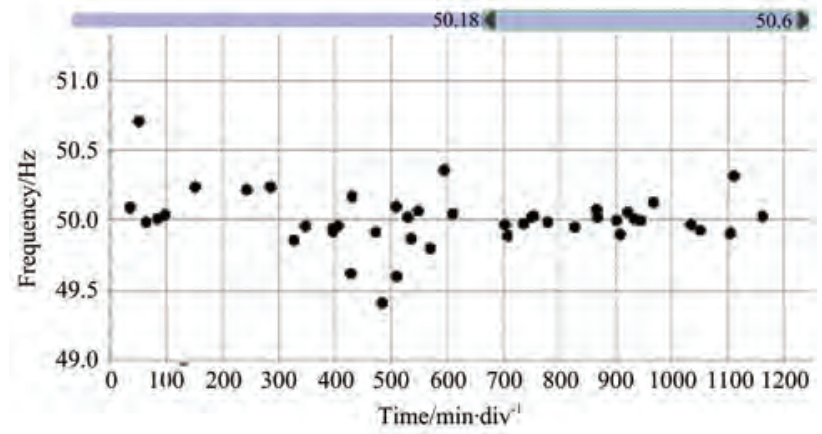

d. Presents time frequency signal in presence of fish in the ranges 50.27-50.29

Figure 9 Signal frequency dispersion in different range in presence of fish

Table 1 Comparison of Measure of Dispersion in Presence and absence of Fish

\begin{tabular}{ccccccc}
\hline Variable & $\begin{array}{c}\text { Biomass } \\
\text { Density/g }\end{array}$ & Observations & Time & RMSE & $\begin{array}{c}\text { Standard } \\
\text { Deviation }\end{array}$ & Sigma2 \\
\hline Frequency 3 & 10000 & 1200 & $5 \mathrm{~min} / \mathrm{div}$ & 230.78 & 0.356 & 9.230 \\
Frequency 2 & 5000 & 1200 & $5 \mathrm{~min} / \mathrm{div}$ & 216.16 & 0.225 & 2.231 \\
Frequency 1 & 0 & 1200 & $5 \mathrm{~min} / \mathrm{div}$ & 204.07 & 0.091 & .009 \\
\hline
\end{tabular}

\subsection{Comparison of frequency signal in different time interval}

In Table 1, Frequency 3 presents the presence with biomass density $1000 \mathrm{~g}$ and Frequency 2 presents the presence with biomass density $500 \mathrm{~g}$ whereas Frequency 1 as in the absence of fish. For each observation division set as $5 \mathrm{~min}$.

\subsection{Machine learning algorithm}

Bayesian regression model was used to show the significant effect of adding fish to the pond. Calculated results of RMSE, Sigma2 in presence of fish $10000 \mathrm{~g}, 5000 \mathrm{~g}$ and in absence of fish respectively shown in Table 1 that significantly distinguishes the effect of add weight to the pond. Receiver operating characteristic (ROC) ${ }^{[37]}$ also proven significant by comparing area under the curve (AUC) for $10000 \mathrm{~g}$ in Figure 10 which is $81 \%$ while AUC for $5000 \mathrm{~g}$ is $71 \%$, against the false positive rate(sensitivity) and true positive rate (1-specificity).

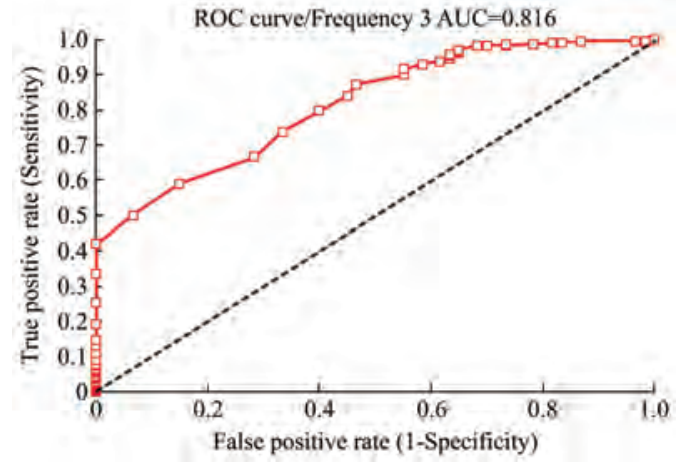

Figure 10 ROC for false positive rate and true positive rate when weight is $10000 \mathrm{~g}$

\subsection{Machine learning algorithm}

Bayesian regression model was used to show the significant effect of adding fish to the pond. Calculated results of RMSE, Sigma2 in presence of fish $10000 \mathrm{~g}, 5000 \mathrm{~g}$ and in absence of fish respectively shown in Table 1 that significantly distinguishes the effect of add weight to the pond. Receiver operating characteristic (ROC) ${ }^{[37]}$ also proven significant by comparing area under the curve (AUC) for $10000 \mathrm{~g}$ in Figure 10 which is $81 \%$ while AUC for $5000 \mathrm{~g}$ is $71 \%$, against the false positive rate(sensitivity) and true positive rate (1-specificity).

Figure 10 and Figure 11 shows the comparison of false positive rate and true positive rate which are the binary classification, as time duration and per minute div of each observation were same, each observation was compared in presence and absence of fish for $10000 \mathrm{~g}$ and $5000 \mathrm{~g}$ in Figure 10 and Figure 11 respectively.

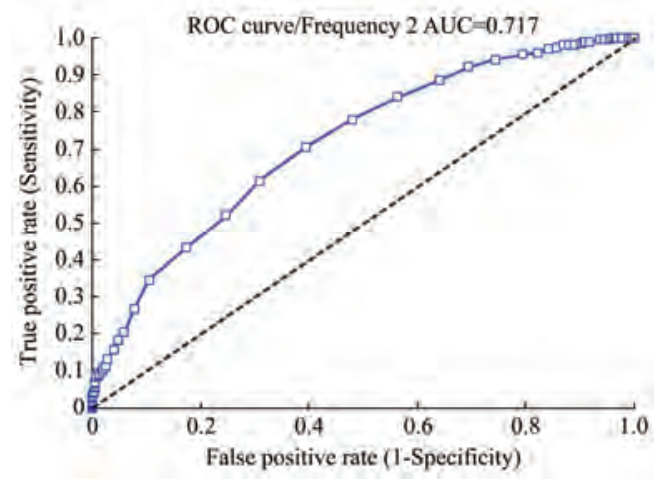

Figure 11 ROC for false positive rate and true positive rate when weight is $5000 \mathrm{~g}$

\section{Conclusions}

For the efficient monitoring system, stable device management and acoustic measurement plays an important role. For this purpose, acoustic device must be protected to create false signals. Moreover, micro-bulls which are created due to high oxygenate 
water must be managed in a way that attenuate the acoustic signals. Since, the acoustic measurement is ratio between the density of tank in presence and absence of fish; it's easy to signify the measure of dispersion between two measurements. Therefore, this study shows that for fish farm or an environment where the external factors are not involved to affect the system, acoustic measurement is highly efficient. Later, it could be integrated with the computer vision to monitor and control more efficiently; especially, in farming system.

The use of acoustic device for proposed method needs determination of limit as expected, when fish have no movement. Presently, the significance difference of acoustic signal in presence and absence of fish by cumulating the signals was measured. For future perspective, it would be interesting to include the feeding factor, depth of acoustic device, effect of temperature. This, in turns will be helpful to measure the growth of fish using acoustic signals.

\section{Acknowledgements}

We acknowledge that this work was financially supported by the Science and Technology Plan of Guangzhou City (Project No. 201807010111), the Science and Technology Plan of Guangdong Province of China (Project No. 2017B090903007) and Innovative Research Team of Guangdong Province Agriculture Research System (2017LM2153) for funding this research. We also thank the anonymous reviewers for their critical comments and suggestions to improve the manuscript. The authors would like to thank a linguistic expert and Dr. Murtaza Hasan for advice when drafting this paper.

\section{[References]}

[1] Cao L, Naylor R, Henriksson P, Leadbitter D, Metian M, Troell M, et al. China's aquaculture and the world's wild fisheries. Science, 2018; 347(6218): 133-135.

[2] Shen X, Chen M, Yu J. In Water environment monitoring system based on neural networks for shrimp cultivation. International Conference on Artificial Intelligence and Computational Intelligence, IEEE, AICI'09, 2009; pp.427-431.

[3] Cromey C, Nickell T, Black K. DEPOMOD - modelling the deposition and biological effects of waste solids from marine cage farms. Aquaculture, 2002; 214(1-4): 211-239.

[4] Ferreira J G, Hawkins A J S, Bricker S B. Management of productivity, environmental effects and profitability of shellfish aquaculture - the Farm Aquaculture Resource Management (FARM) model. Aquaculture, 2007; 264(1-4): 160-174.

[5] Pérez O, Telfer T, Beveridge M. Geographical Information Systems (GIS) as a simple tool to aid modelling of particulate waste distribution at marine fish cage sites. Estuarine, Coast, 2002; 54(4): 761-768.

[6] Corner R, Brooker A, Telfer T, Ross L. A fully integrated GIS-based model of particulate waste distribution from marine fish-cage sites. Aquaculture, 2006; 258(1-4): 299-311.

[7] Li D L, Hassan M, Khan K A, Abbas A, Bano S. Design and instrumentation of Portable Monitoring System for acoustic measurement based on aquatic density. 2017 IEEE International Conference on Computational Science and Engineering (CSE) and Embedded and Ubiquitous Computing (EUC), 2017; 2: pp.166-172.

[8] Huettmann F. Towards a Marine Environmental Information System (MEnvIS) for the Northwest Atlantic: Experiences and suggestions from a multi-disciplinary GIS. Fourth World Sci. Eng. Acad, 2003; 1: 82-90.

[9] McCausland W, Mente E, Pierce G. A simulation model of sustainability of coastal communities: aquaculture, fishing, environment and labour markets. Ecological Modelling, 2006; 193(3-4): 271-94.

[10] Deshmukh S G, Nitin U, Suresh G. Lean manufacturing system for medium size manufacturing enterprises: an Indian case. International Journal of Management Science and Engineering Management, 2010; 5(5):
$362-75$

[11] MEDVEG. http://www.ist-world.org/Project Details.aspx? ProjectId= eddefd9a8e4248a6b0662fa2d33af423. Accessed on [2010-11-01]

[12] MERAMED. http://meramed.akvaplan.com/, Accessed on [2010-11-01]

[13] ICES Working Group on Environmental Interactions of Mariculture http://www.ices.dk/workinggroups/ViewWorkingGroup.aspx?ID=165. Accessed on [2010-11-01]

[14] EACASA. http://www.ecasa.org.uk/. Accessed on [2010-11-01]

[15] Mauri G, Mauri J. Simulator software for marine fish farms sustainability. WSEAS Transactions on Environment and Development, 2007; 3(12): 214-22.

[16] Zelick R, Mann D A, Popper A N. Acoustic communication in fishes and frogs. In Comparative hearing: Fish and amphibians, Springer, 1999; 363-411.

[17] Popper A N. Effects of anthropogenic sounds on fishes. Fisheries, 2003; 28(10): 24-31.

[18] Baras E, Lagardère J P. Fish telemetry in aquaculture: Review and perspectives. Aquaculture International, 1995; 3(2): 77-102.

[19] Baras E. Selection of optimal positioning intervals in fish tracking: An experimental study on barbus barbus. In Advances in invertebrates and fish telemetry, Springer, 1998; pp.19-28.

[20] Van den Acker E, Van Hoolst T, de Viron O, Defraigne P, Forget F, Hourdin $\mathrm{F}$, et al. Influence of the seasonal winds and the $\mathrm{CO}_{2}$ mass exchange between atmosphere and polar caps on mars' rotation. Journal of Geophysical Research: Planets, 2002; 107(E7): 1-8.

[21] Juell J, Furevik D, Bjordal Å. Demand feeding in salmon farming by hydroacoustic food detection. Aquacultural Engineering, 1993; 12(3): $155-167$.

[22] Anras M B, Covés D, Dutto G, Laffargue P, Lagardère F. Tagging juvenile seabass and sole with telemetry transmitters: Medium-term effects on growth. ICES Journal of Marine Science: Journal du Conseil, 2003; 60(6): 1328-1334.

[23] De Rosny J, Roux P. Multiple scattering in a reflecting cavity: Application to fish counting in a tank. The Journal of the Acoustical Society of America, 2001; 109(6): 2587-2597.

[24] Hassan S G, Hasan M. Information fusion in aquaculture: A state-of the art review. Frontiers of Agricultural Science and Engineering, 2016; 3(3): 206-21.

[25] De Rosny J, Roux P, Fink M, Page J. Field fluctuation spectroscopy in a reverberant cavity with moving scatterers. Physical review letters, 2003; 90(9): 094302.

[26] Demer D A, Conti S G. Validation of the stochastic distorted-wave born approximation model with broad bandwidth total target strength measurements of antarctic krill. ICES Journal of Marine Science: Journal du Conseil, 2003; 60(3): 625-635.

[27] Demeret C, Garcia-Carranca A, Thierry F. Transcription-independent triggering of the extrinsic pathway of apoptosis by human papillomavirus 18 e2 protein. Oncogene, 2003; 22(2): 168 .

[28] Conti S G, Demer D A, Brierley A S. Broad-bandwidth, sound scattering, and absorption from krill (meganyctiphanes norvegica), mysids (praunus flexuosus and neomysis integer), and shrimp (crangon crangon). ICES Journal of Marine Science: Journal du Conseil, 2005; 62(5): 956-965.

[29] Alanärä A, Burns M D, Metcalfe N B. Intraspecific resource partitioning in brown trout: The temporal distribution of foraging is determined by social rank. Journal of Animal Ecology, 2001; 70(6): 980-986.

[30] Jobling M. Do changes in atlantic salmon, salmo salar 1., fillet fatty acids following a dietary switch represent wash - out or dilution? Test of a dilution model and its application. Aquaculture Research, 2003; 214(1-4): 211-239.

[31] Mallekh R, Lagardere J, Eneau J, Cloutour C. An acoustic detector of turbot feeding activity. Aquaculture, 2003; 221(1-4): 481-489;

[32] ummerfelt $\mathrm{S} \mathrm{T}$, Holland $\mathrm{K} \mathrm{H}$, Hankins J A., Durant $\mathrm{M}$ D. A hydroacoustic waste feed controller for tank systems. Water Science and Technology 1995; 31(10): 123-129.

[33] Juell J E. Hydroacoustic detection of food waste - a method to estimate maximum food intake of fish populations in sea cages. Aquacultural Engineering, 1991; 10(3): 207-217.

[34] Lagardère J, Mallekh R. Feeding sounds of turbot (scophthalmus maximus) and their potential use in the control of food supply in aquaculture: I. Spectrum analysis of the feeding sounds. Aquaculture, 2000; 189(3-4): 251-258. 
[35] Rogers P H, Cox M. Underwater sound as a biological stimulus. In Sensory biology of aquatic animals, Springer: 1988; pp.131-149.

[36] Kalmijn A J. Functional evolution of lateral line and inner ear sensory systems. In the Mechanosensory Lateral Line, Springer: 1989; pp.187-215.
[37] Viejo C G, Fuentes S, Howell K, Torrico D, Dunshea F R. Robotics and computer vision techniques combined with non-invasive consumer biometrics to assess quality traits from beer foamability using machine learning: A potential for artificial intelligence applications. Food control, 2018; 92: 72-79. 